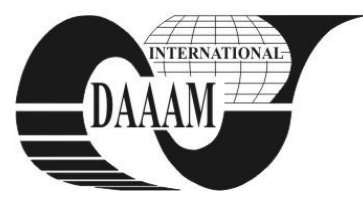

Annals of DAAAM for 2011 \& Proceedings of the 22nd International DAAAM Symposium, Volume 22, No. 1, ISSN 1726-9679 ISBN 978-3-901509-83-4, Editor B. Katalinic, Published by DAAAM International, Vienna, Austria, EU, 2011 Make Harmony between Technology and Nature, and Your Mind will Fly Free as a Bird Annals \& Proceedings of DAAAM International 2011

\title{
AUTOMATED RESERVE ARMING CONTROL SYSTEM FOR POWER SUPPLY
}

\author{
BADEA, M[ilian]; MORARU, S[orin] - A[urel] \& GRIGORESCU, C[ostin] - M[arius]
}

\begin{abstract}
This paper presents an application that can be used to maintain the power supply for industrial consumers. The power supply is maintained by monitoring the presence or absence of voltage on power lines and selecting the corresponding one to provide electrical energy for the system's consumers. The monitoring and control system is composed of a Siemens 315 PLC, a Siemens MP 277 touch screen operating panel and a series of Sentron PAC3200 power monitoring devices that communicate with each other using Profibus, MPI and Modbus networks and communication protocols.

Key words: monitoring device, energy, power, modbus, profibus
\end{abstract}

\section{INTRODUCTION}

The high reliability of electrical energy supply of industrial processes is a very important issue because it can prevent the losses and damages that can be produced by intermittent electrical energy supply. In order to achieve this, the supply with electrical energy of the industrial consumers can be made using different ways and different equipment.

The application presented in this paper makes the Automated Reserve Arming (ARA) in the electrical energy supply system of a commercial unit. This supply system consists of two separate supply lines and two energy generators. Switching between the supply lines and the energy generators is made in automatic manner in order to provide almost continuous electrical energy supply (Margineanu, 2005, Vladareanu, 2005).

\section{SYSTEM STRUCTURE}

The Automated Reserve Arming project is made with the help of the following hardware equipment:

- A Siemens CPU 315-2-DP PLC.

- A Siemens MP 277 Operating Panel.

- A series of Sentron PAC3200 power monitoring devices.

The structure of the entire system is presented in Fig. 1 and the roles of the equipment are presented in the following paragraphs.

The Siemens MP 277 Operating Panel is used to display the parameters of the electrical energy supply network and communicates with the PLC unit through a MPI network.

The Siemens Sentron PAC3200 power monitoring devices communicate with the PLC unit through a Profibus network. These devices monitor a number of network parameters and send them to the PLC unit (Margineanu, 2005, Popescu, D., 2005). These measurements are very useful for the human operator because they show exactly all the system state changes that appear while the system is in operation.

The information from the power generators is received through a RS485 network using the Modbus communication protocol (Popescu, S., 2005).

The supply with electrical energy is made through two separate transformers, one being primary and the other the reserve. The system also uses two generators that can provide the needed electrical energy in the case of voltage lask on both transformer lines. Switching between the reserve and the generators is made using automated arming switches.

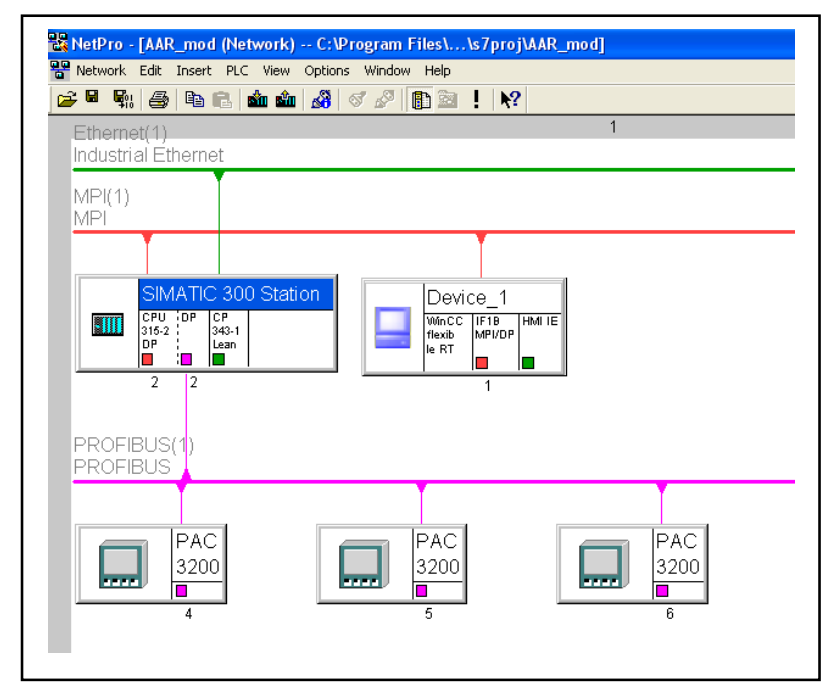

Fig. 1. System structure

The state of these switches is read using the PLC's digital inputs and the command to open or close one is made using the PLC's digital outputs. The protection against simultaneous closing of both line switches is implemented at both hardware and software levels.

Switching between the supply lines is made based on the presence or absence of voltage on them. To verify the presence or absence of the three voltage phases on the supply lines the following methods are implemented:

- Using relays that detect the presence or absence of voltage.

- Checking the values provided by the three Siemens Sentron PAC3200 power monitoring devices.

\section{APPLICATION PRESENTATION}

Three operating modes were implemented for the application. These modes are:

- Manual.

- Semi automatic.

- Full automatic.

The manual operating mode allows the human operator to close or open any switch. The hardware level protection is still present.

The semi automatic operating mode consists of making a complete switching operation from one power source to another one. The start of the sequence is made using a button from the system's operating panel.

The sequence consists of the following steps:

- Opening the already closed switch. 
- Making a temporization to wait for the electric arc to disappear.

- Closing the switch for the desired power source.

The full automatic operating mode represents the normal operating mode of the system. The network is monitored by the Sentron devices and the voltage detection relays.

The transformer that powers the consumers when the system goes into this operating mode is considered to be the main one. It will power the consumers as long as the relays detect the presence of voltage on its lines.

If, for any reason, the voltage is not detected then the system will wait for 2 seconds to avoid switching in the case of very short power failures and after that time it will make the switch to the second transformer which is stored as the reserve one.

If the voltage is absent also on the lines of the second transformer, then the backup generators will be powered on. When the voltage is present again, the power source is chosen using the following criteria:

- If the voltage is present on the lines of both transformers, then the system will select the main transformer.

- If the voltage is present on the lines of the reserve transformer, then this one will be selected.

In both cases, the backup generators will be powered off.

The human operator can view the real time value of the main system perameters on the operating panel. From the defined screens, the most important one is represented by the single line diagram screen presented in Fig. 2 (Simatic HMI MP 277 Operating Instructions, 2009).

This screen presents the status of the switches, the presence or absence of voltage on different sections of the power lines and the values of the power network parameters received from the Sentron PAC3200 power monitoring devices. These values are received through the Profibus network.

The elements that are presented with the red color on this screen are currently powered on and those presented with black are currently powered off.

The variations and events are treated separately. They are stored in a history location that can keep up to 200.000 records. If this value is exceded, then the oldest records are erased to allow the new ones to be stored. The events are stored in order to be able to replay some sequences of the ARA system (Springer, 2006).

The alarms are represented by actions that can endanger the system's operation and need to be treated urgently. They block the ARA system in its current state and don't allow for any switching operation until the human operator acknowledges them by pressing a Reset button.

The alarms are signaled both visual and acoustic.

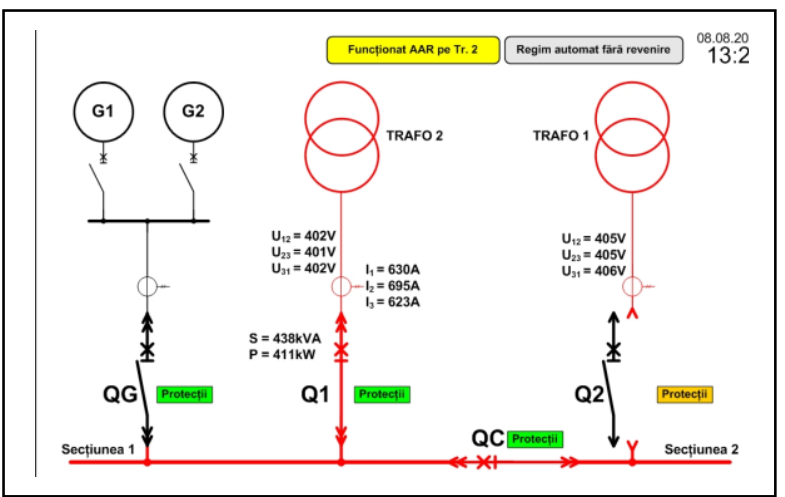

Fig. 2. Single line diagram screen

\section{MODBUS COMMUNICATION}

The communication with the generators is made using the Modbus protocol. The CP341 communication processor of the
Siemens PLC implements only the ASCII version of the Modbus protocol (CP341: Point-to-Point Communication, Installation and Parameter Assignment, 2000). In order to be able to use the RTU version of the same protocol, the users must purchase some hardware and software items from the PLC's manufacturer (Loadable Driver for Point-to-Point CPs, Modbus Protocol, RTU Format, 2003).

In order to be able to communicate with Modbus RTU slave units using the ASCII version, the following steps needed to be made:

- A CRC16 calculation method was implemented.

- When sending an ASCII message, the two CRC16 checksum bytes were added at the end of it.

- When receiving an ASCII message, the two CRC16 checksum bytes are extracted and for the remaining message the CRC16 checksum is calculated.

- The calculated CRC16 checksum and the received one are compared.

If the munber of bytes received is equal with the one requested and if the two checksums are equal, then the communication has ended without any errors and a new reading can be initiated.

\section{CONCLUSION}

An Automated Reserve Arming control system used for providing continuous electrical energy supply for industrial consumers is very useful because industrial processes depend on reliable electrical energy supply.

This system can also be used to monitor the quality of the power supply by using the measured parameters provided by the Sentron PAC3200 power monitoring devices. These devices have the following main features: direct voltage measurement on the system or using voltage transformers; monitors more than 50 parameters; offers 10 energy counters to capture active energy, reactive energy, apparent energy for off - peak and on - peak, etc.

\section{ACKNOWLEDGEMENTS}

This paper is supported by the Sectoral Operational Programme Human Resources Development, financed from the European Social Fund and by the Romanian Government under the project number POSDRU/107/1.5/S/76945.

\section{REFERENCES}

Margineanu, I. (2005). Automate programabile, Ed. Albastra, ISBN 973-650-156-6

Popescu, D. (2005). Automate programabile. Constructie, functionare, programare si aplicatii, Ed. Matrix Rom Bucuresti, ISBN 973-685-942-8

Popescu, S. (2005). Tehnici avansate de comunicatie, Ed. Albastra, ISBN 973-650-127-2

Springer, B. (2006). A Monitoring System for PLC Controlled Manufacturing System Based on Fieldbus, Knowledge Enterprise: Intelligent Strategies in Product Design, Manufacturing and Management 207

Vladareanu, L. (2005), Controlul in timp real cu automate programabile in mecanica solidului. Studii si cercetari aplicative, Ed. Bren, ISBN 973-648-432-7

*** (2000) CP341: Point-to-Point Communication, Installation and Parameter Assignment, Siemens

*** (2003) Loadable Driver for Point-to-Point CPs, Modbus Protocol, RTU Format, Siemens

*** (1996) Modbus Protocol Reference Guide, Modicon

*** (2009) Simatic HMI MP 277 Operating Instructions, Siemens 